\title{
Supplémentation en céréales, état corporel et enzymes lipogéniques du tissu adipeux chez la brebis Aragonesa
}

\author{
I Sebastian 1,2, Y Chilliard $2,{ }^{*}$, A Purroy ${ }^{3, *}$, C Jaime 3
}

avec la collaboration technique de $\mathrm{J}_{\text {Flechet }}{ }^{2}, \mathrm{~F}$ Lahoz $^{3}, \mathrm{E}$ Morago ${ }^{3}$

\author{
'ETSEAL-UPC, Sección de Producción Animal, Rovira Roure 177, 25006 Lerida, Espagne; \\ 2 INRA-Theix, laboratoire de recherches sur la Sous-Nutrition des ruminants, \\ 63122 Saint-Genès-Champanelle, France; \\ 3 SIA (DGA), Unidad de Producción Animal, Apdo 727, 50080 Zaragoza, Espagne;
}

(Reçu le 28 février 1992; accepté le 21 décembre 1992)

\begin{abstract}
Résumé - Dix-huit brebis adultes de race Aragonesa ont reçu de la paille traitée à l'ammoniaque ad libitum, supplémentée soit par $250 \mathrm{~g}$ (lot B), soit par $500 \mathrm{~g}$ (lot H) d'orge par j, pendant une période de $10 \mathrm{sem}$ après le sevrage des agneaux. L'ingestion et la digestibilité de la ration ont été mesurées. Du tissu adipeux mésentérique a été prélevé par biopsie 5 j et 10 sem après le début de l'expérience. La réduction de la supplémentation en orge a provoqué une augmentation de l'ingestion de paille (1 217 vs $961 \mathrm{~g} / \mathrm{j})$ ), sans modifier l'ingestion de matière sèche totale (1450 vs $1430 \mathrm{~g} /$ j). Elle a aussi diminué légèrement ( -4 points) et non significativement la digestibilité de la matière organique de la ration. L'ingestion d'énergie a été légèrement plus faible chez les brebis du lot $B$ (92 vs $100 \mathrm{kcal}$ énergie nette/j/kg $P V 0,75, P<0,05)$. Le niveau de supplémentation en orge n'a modifié ni le gain de poids vif (environ $7 \mathrm{~kg}$ ), ni l'accroissement de la note d'état corporel (environ 0,5 point), ni l'augmentation du volume moyen des adipocytes du tissu adipeux mésentérique (environ $190 \mathrm{pl}$ ) ou de sa teneur en lipides (environ $24 \mathrm{~g} / 100 \mathrm{~g}$ ). Le volume moyen des adipocytes est corrélé avec la note d'état corporel des brebis $(r=0,79, n=36)$ ainsi qu'avec la quantité totale de lipides du tissu adipeux mésentérique estimée à partir de la note d'état corporel et de la teneur en lipides de ce tissu $(r=0,81)$. L'augmentation du niveau de supplémentation en orge (lot $\mathrm{H})$ a entraîné un accroissement significatif des activités de l'enzyme malique, de la glycérol-3-phosphate déshydrogénase (G3PDH) et de la glucose-6-phosphate déshydrogénase au j 5. En revanche, à la fin de la période expérimentale (sem 10), seule l'activité de la G3PDH est significativement plus élevée chez les animaux du lot $H$. Les activités de la synthétase d'acides gras et de la lipoprotéine-lipase n'ont pas varié entre les 2 lots et les 2 périodes. Cette étude montre donc que la diminution de 500 à $250 \mathrm{~g} / \mathrm{j}$
\end{abstract}

\footnotetext{
* Correspondance et tirés à part

** Adresse actuelle : ETSIA, Departamento de Producción Agraria, Universidad Pública de Navarra,

El Sadar s/n, 31006 Pamplona, España
} 
de l'apport d'orge ne modifie pas significativement la reconstitution des réserves corporelles ni les activités lipogéniques du tissu adipeux mésentérique.

brebis / paille / orge / état corporel / volume des adipocytes / enzymes lipogéniques / tissu adipeux mésentérique

Summary - Effects of the supplementation of a straw-based diet with concentrate on body condition and lipogenic activity of the mesenteric adipose tissue in the Aragonesa ewe after weaning. Eighteen Aragonesa adult ewes were fed ammonia-treated straw ad libitum supplemented with 250 (group L) or $500 \mathrm{~g} / \mathrm{d}$ (group H) barley over a 10-wk period following weaning. Feed consumption and diet digestibility were measured. $A$ biopsy of mesenteric adipose tissue was carried out $5 d$ and 10 wk after the start of the experiment.

The reduced barley supplementation increased straw dry matter (DM) intake $(1217 \mathrm{vs} 961 \mathrm{~g} / \mathrm{d})$, had no effect on total DM intake (1 450 vs $1430 \mathrm{~g} / \mathrm{d}$ ), and slightly reduced ( -4 points) organic matter digestibility of the diet. Energy intake was slightly lower in group L than in group H (92 vs $100 \mathrm{kcal}$ net energy/d/kg BW0.75, respectively).

The level of barley supplementation had no effect on body weight gain $(\approx 7 \mathrm{~kg})$, increase in body condition score $(\approx 0.5$ point), increase in mean volume $(\approx 190 \mathrm{pl})$ of the mesenteric adipose cells, or lipid concentration in the latter $\approx 24 \mathrm{~g} / 100 \mathrm{~g})$. The mean volume of the adipose cells was correlated with body condition score $(r=0.79, \mathrm{n}=36)$, and also with the amount of lipid in the mesenteric adipose tissue as estimated from the body condition score and the lipid content of this tissue $(r=0.81)$.

Compared with that of group $L$, the adipose tissue of group $H$ showed higher malic enzyme, glycerol3-phosphate dehydrogenase (G3PDH) and glucose-6 phosphate dehydrogenase activity at d 5. At wk 10, G3PDH was the only enzyme whose activity remained higher in group $H$. Fatty acid synthetase and lipoprotein lipase activity did not differ between supplementation levels or experimental periods. It is concluded that the reduced barley supplementation from 500 to $250 \mathrm{~g} / \mathrm{d}$ did not significantly alter reconstitution of the body reserves or the lipogenic activity of the mesenteric adipose tissue.

sheep / straw / barley / body condition / adipose cell volume / lipogenic enzyme / mesenteric adipose tissue

\section{INTRODUCTION}

L'intensité de la mobilisation des réserves corporelles lipidiques et les variations de l'activité métabolique du tissu adipeux ont surtout été étudiées chez la brebis pendant les périodes de bilan énergétique négatif en fin de gestation et durant la lactation (Russel et al, 1968; Cowan et al, 1980 ; Vernon et al, 1981, 1986, 1987 ; Bocquier et al, 1987b; Chilliard, 1987 ; Guesnet et al, 1991). Cependant, peu d'études (Hood et Thornton, 1980 ; Smith et Walsh, 1984) ont concerné les périodes de bilan énergétique positif en fin de lacta- tion et surtout après le sevrage des agneaux, lorsque les brebis reconstituent les réserves lipidiques qu'elles ont précédemment mobilisées.

Une pratique habituelle dans l'élevage de type extensif des brebis est l'utilisation de fourrages grossiers peu coûteux, comme la paille d'orge traitée à l'ammoniaque, durant les périodes où les besoins sont faibles. Toutefois, si la mobilisation lipidique a été intense pendant la lactation, il est nécessaire de complémenter ces fourrages avec des quantités variables de céréales, afin de permettre la reconstitution des réserves lipidiques avant la mise en reproduction. 
Dans un essai précédent (Sebastián et al, 1989), la récupération postsevrage a été étudiée pendant $80 \mathrm{j}$ avec une ration de paille traitée ad libitum et $400 \mathrm{~g} / \mathrm{j}$ d'orge en moyenne. II est apparu que des brebis rustiques de race Rasa Aragonesa, initialement maigres, ont accru leur poids vif et leur note d'état corporel, mais que cela ne s'est pas accompagné d'un accroissement de la taille moyenne des adipocytes du tissu adipeux sous-cutané.

L'objectif du présent essai est de rechercher si la récupération postsevrage des réserves corporelles s'accompagne de modifications de la taille et des activités d'enzymes lipogéniques des adipocytes dans un autre site anatomique (tissu adipeux mésentérique), et si cette récupération est sensible à une variation de la supplémentation en orge (250 vs $500 \mathrm{~g} / \mathrm{j})$ d'une ration à base de paille traitée à l'ammoniaque.

\section{MATÉRIEL ET MÉTHODES}

\section{Animaux et traitements}

Dix-huit brebis adultes de race Aragonesa (RA) ont été conduites pendant la fin de gestation et 62 jours de lactation d'une manière voisine à celle qui a été décrite pour un essai précédent (Sebastián et al, 1989). Après le sevrage des agneaux, les brebis ont été réparties en 2 lots de 9 , équilibrés selon le poids vif et la note d'état corporel. Elles ont reçu une ration à base de paille traitée à l'ammoniaque ( $35 \mathrm{~g} \mathrm{NH}_{3}$ anhydre/kg MS) et complémentée soit par $250 \mathrm{~g}$ (lot B), soit par $500 \mathrm{~g}$ (lot H) d'orge par jour, pendant une période expérimentale de $11 \mathrm{sem}$.

La quantité de paille traitée offerte a été augmentée progressivement (de 700 à $1000 \mathrm{~g} / \mathrm{j}$ ) pendant la première semaine, puis distribuée ad libitum de façon à obtenir un taux de refus d'environ $10 \%$. La paille et l'orge correspondant à la ration journalière ont été mélangés et distribués en 2 parties égales à 9 et à $14 \mathrm{~h}$. Les quantités ingérées ont été mesurées chaque jour, et la te- neur en matière sèche (MS) de la paille offerte et refusée a été mesurée une fois par sem sur un échantillon moyen représentatif.

Le poids vif et la note d'état corporel des brebis ont été mesurés au début et à la fin de la période expérimentale. La note d'état corporel a été appréciée par palpation au niveau lombaire, sur une échelle de 0 à 5 (Russel et al, 1969).

\section{Digestibilité et valeur énergétique de la ration}

La digestibilité de la MS et de la matière organique ont été mesurées sur 4 brebis pour chacun des 2 types de ration pendant une période de $8 \mathbf{j}$ à la fin de la période expérimentale (sem 11).

Les fèces de chaque animal ont été recueillies et leur MS déterminée quotidiennement. Les analyses ultérieures ont été réalisées sur un échantillon moyen représentatif de la période de mesure pour chaque brebis. La MS des aliments offerts et refusés et des fèces a été déterminée à l'étuve à $80^{\circ} \mathrm{C}$ pendant $48 \mathrm{~h}$ et la teneur en cendres après incinération à $550^{\circ} \mathrm{C}$ pendant $3 \mathbf{h}$.

L'énergie totale ingérée a été calculée en kcal d'énergie nette de lactation (ENI) à partir des quantités d'aliments ingérés, de la digestibilité mesurée de la ration totale et des équations proposées par l'INRA (1988). On a estimé aussi le bilan énergétique, correspondant à l'énergie ingérée au-dessus du besoin d'entretien, soit sur l'ensemble de la période expérimentale (bilan moyen des sem 2 à 10), soit pour les 2 sem (sem 1 et 10) durant lesquelles ont été effectués les prélèvements de tissu adipeux.

\section{Prélèvement du tissu adipeux et determination de sa composition chimique et de la taille des adipocytes}

$\dot{A}$ la fin des sem 1 et 10 , alors qu'elles avaient été immobilisées pour réaliser une endoscopie ovarienne, une biopsie a été effectuée sur les 18 brebis pour prélever un échantillon de 5 à 10 $\mathrm{g}$ de tissu adipeux mésentérique. Une petite fraction de cet échantillon (200-500 mg) a été conservée dans du sérum physiologique à $39^{\circ} \mathrm{C}$ afin de mesurer la taille des adipocytes. Le reste 
de l'échantillon a été transporté immédiatement sur de la glace au laboratoire, où des parties aliquotes $(0,75$ à $1,5 \mathrm{~g}$ environ) exemptes de sang et de tissu conjonctif ont été préparées et congelées à $-20^{\circ} \mathrm{C}$ jusqu'à la détermination de la composition chimique et des activités enzymatiques.

La taille moyenne des adipocytes a été déterminée après fixation du tissu par l'acide osmique (Robelin, 1981 ; Robelin et Barboiron, 1988). Afin de réduire la surestimation du volume moyen des adipocytes due à l'utilisation de cette méthode sur des animaux très maigres (Sebastián et al, 1989), on a pris en compte toutes les cellules d'un diamètre supérieur à 5 microns, en utilisant un grossissement de 200 au lieu de 80 pour la photographie sous microscope.

La MS du tissu adipeux a été mesurée par lyophilisation pendant $48 \mathrm{~h}$ sur un échantillon d'environ $1 \mathrm{~g}$. Les lipides totaux ont été extraits par 2 homogénéisations successives d'environ $150 \mathrm{mg}$ de tissu adipeux dans $6 \mathrm{ml}$ d'heptane (broyeur Polytron, 25000 tours/min, pendant $30 \mathrm{~s}$ ) suivies par 2 centrifugations ( $3000 \mathrm{~g}$ pendant 20 min à $4^{\circ} \mathrm{C}$ ), et déterminés par pesée.

Le nombre de cellules par gramme de tissu a été calculé d'après le volume moyen des cellules et la teneur en lipides du tissu adipeux, en considérant que la densité des lipides de l'adipocyte est en moyenne de 0,915 (Robelin, 1981).

\section{Activité des enzymes lipogéniques du tissu adipeux}

Les activités de la glucose-6-phosphate déshydrogénase (G6PDH, EC 1.1.1.49), de la NADPmalate déshydrogénase (enzyme malique, $E M$, EC 1.1.1.40), de la glycérol-3-phosphate déshydrogénase (G3PDH, EC 1.1.1.8) et de la synthétase d'acides gras (Fatty acid synthetase, FAS, EC 2.3.1.85) ont été déterminées par spectrophotométrie sur des homogénats de tissu adipeux mésentérique.

Pour l'extraction de ces 4 enzymes, on a utilisé des échantillons congelés d'environ $1 \mathrm{~g}$, découpés finement au scalpel puis homogénéisés dans $4 \mathrm{ml}$ d'un milieu saccharose $(300 \mathrm{mM})$; Tris $(30 \mathrm{mM}, \mathrm{pH} 7,4)$, EDTA (1 mM) et glutathion (1 mM), à l'aide d'un microhomogénéiseur
(Sorvall Omni Mixer 17106, Norwalk, USA) à la vitesse de rotation de 50000 tours/min pendant 6 périodes de $10 \mathrm{~s}$ espacées par 5 périodes de $10 \mathrm{~s}$ de refroidissement $\left(0-4^{\circ} \mathrm{C}\right)$. Les homogénats ont ensuite été filtrés sur un tissu de Nylon (maille de 10 microns), de façon à éliminer les débris tissulaires et la majeure partie des lipides, puis centrifugés (Centrikon $\mathrm{H}-401$, Kontron) pendant $45 \mathrm{~min}$ à $20000 \mathrm{~g}$ à $4^{\circ} \mathrm{C}$. La fraction cytosolique a été recueillie à l'aide d'un tube capillaire, et conservée à $4^{\circ} \mathrm{C}$ jusqu'au dosage dans les 3-4 h suivantes, ou bien congelée à $-80^{\circ} \mathrm{C}$ pour des dosages ultérieurs.

L'activité de la G6PDH a été mesurée d'après la méthode de Glock et McLean (1953) par incubation de 20 et $40 \mu \mathrm{l}$ d'homogénat dans un milieu constitué de tampon glycylglycine ( 100 $\mathrm{mM}, \mathrm{pH} 7,6)$, de chlorure de magnésium (10 $\mathrm{mM})$, de NADP+ $(0,2 \mathrm{mM})$ et de glucose-6phosphate (2 mM) (volume final de 3,2 $\mathrm{ml}$ ).

L'activité de l'EM a été mesurée d'après la méthode d'Ochoa (1955) par incubation de 20 et $40 \mu \mathrm{l}$ d'homogénat dans un milieu contenant du tampon triéthanolamine $(66 \mathrm{mM}, \mathrm{pH} 7,4)$, du chlorure de manganèse ( $4 \mathrm{mM})$, du NADP+ $(0,2$ $\mathrm{mM}$ ) et du malate $(0,5 \mathrm{mM})$ (volume final de 3,0 $\mathrm{ml}$ ).

L'activité de la G3PDH a été mesurée d'après la méthode de Wise et Green (1979) avec 30 et $60 \mu \mathrm{l}$ d'homogénat (préalablement dilué de 10 à 30 fois, dans le tampon d'extraction) dans une solution de triéthanolamine (100 mM, pH 7,5), d'EDTA (3 mM), de NADH $(0,15 \mathrm{mM})$ et de dihydroxyacétone-phosphate $(1,2 \mathrm{mM})$ (volume final de $3,1 \mathrm{ml}$ ).

L'activité de la FAS a été mesurée d'après la méthode de Halestrap et Denton (1973) par incubation de 40 et $80 \mu$ l'homogénat dans une solution de phosphate de potassium $(100 \mathrm{mM}$, $\mathrm{pH} 6,8)$, d'EDTA $(1 \mathrm{mM})$, de dithiothreitol $(1 \mathrm{mM})$, de NADPH $(0,15 \mathrm{mM})$, d'acétyl-CoA $(0,05 \mathrm{mM})$ et de malonyl-CoA $(0,1 \mathrm{mM})$ (volume final de $3,1 \mathrm{ml}$ ).

La cinétique de la réaction a été enregistrée chaque minute pendant 10 à 20 min selon l'enzyme, par différence avec un blanc échantillon sans substrat (spectrophotomètre à double faisceau Uvikon 810; Kontron). L'activité spécifique de chaque enzyme a été calculée à partir de la mesure de la quantité de NADPH apparue (G6PDH et EM) ou de la quantité disparue de NADH (G3PDH) ou de NADPH (FAS) à une longueur d'onde de $340 \mathrm{~nm}$, en utilisant les 4 va- 
leurs les plus élevées de variation de densité optique par minute. Les activités ont été exprimées en $\mu$ moles de nucléotides oxydés ou réduits par $\mathrm{h}$ et par $\mathrm{g}$ de tissu adipeux, ou par million d'adipocytes.

L'activité de la lipoprotéine-lipase (LPL, EC 3. 1.1.34) a été mesurée (Chilliard et al, 1977, 1991) après extraction par homogénéisation du tissu dans du tampon ammoniacal $(50 \mathrm{mM}$, $\mathrm{pH} 8,3$ ) additionné de 5,6 unités/ml d'héparinate de sodium. Après filtration sur tissu de Nylon (maille 10 microns), l'homogénat $(1 \mathrm{ml})$ a été incubé pendant 45 et $90 \mathrm{~min}$, dans un milieu (volume final de $2,5 \mathrm{ml}$ ) contenant du tampon Tris (120 mM, pH 8,3), de la sérum-albumine bovine (fraction $\mathrm{V}, 4 \%$, p:v), une émulsion commerciale (Intralipide, Vitrum) de triglycérides (0,33\%, p:v) préalablement activée $\left(45 \min\right.$ à $37^{\circ} \mathrm{C}$ ) par 4 volumes de sérum sanguin bovin (concentration finale $13,3 \%, v: v)$ et du chlorure de sodium (54 mM). La quantité d'acides gras libérés pendant l'incubation a été déterminée par titrimétrie, et les résultats exprimés en $\mu$ moles d'acides gras libérés par heure et par gramme de tissu adipeux, ou par million d'adipocytes.

Tous les produits utilisés ont été fournis par Sigma (La Verpillère, France) l'exception de la glycylglycine et de la triéthanolamine (Merck, Darmstadt, Allemagne).

\section{Analyses statistiques}

Les différences entre traitements ont été analysées par le test $t$ de Student, et les variations entre les sem 1 et 10 par la méthode des couples (test $t$ apparié).

\section{RÉSULTATS}

\section{Ingestion et digestibilité}

Conformément au protocole d'alimentation en début d'expérience, l'ingestion de matière sèche totale a atteint des valeurs moyennes maximales de 1550 et $1570 \mathrm{~g} /$ $\mathrm{j} /$ animal dans les lots $\mathrm{H}$ et $\mathrm{B}$, respectivement, entre les semaines 4 et 5 . Ultérieu- rement, l'ingestion a eu tendance à diminuer légèrement puis à se stabiliser jusqu'à la sem 10 (fig 1). La réduction du niveau d'orge distribué en complémentation de la paille a entraîné une augmentation de la quantité de paille ingérée (fig 1), ce qui explique que l'ingestion de matière sèche (totale ou par $\mathrm{kg}$ de $\mathrm{PV}^{0,75}$ ) ait été voisine dans les 2 lots.

La réduction du niveau d'orge n'a diminué que légèrement ( -4 points), et de façon non significative $(P>0,10)$, les digestibilités de la MS et de la matière organique de la ration mesurées à la fin de la période expérimentale (tableau I).

L'ingestion plus élevée de paille dans le lot $B$ (valeurs moyennes des semaines 2 à 10 de 68 et $53 \mathrm{~g} / \mathrm{j} / \mathrm{kg}$ PV 0,75 pour les lots B et $H$, respectivement) (tableau II) n'a toutefois pas entièrement compensé la différence d'apport énergétique entraînée par l'ingestion plus faible d'orge : l'ingestion d'énergie a été légèrement plus faible dans le lot $\mathrm{B}$ que dans le lot $\mathrm{H}(P<0,05)$. La différence a été plus importante pendant la

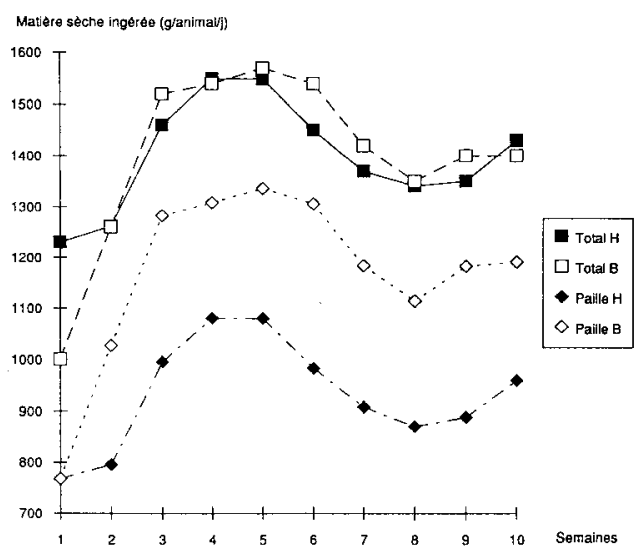

Fig 1. Évolution de l'ingestion de matière sèche pendant la période expérimentale. Les carrés fermés et ouverts représentent l'ingestion de la ration totale, et les losanges fermés et ouverts l'ingestion de paille pour les régimes $\mathrm{H}$ et $\mathrm{B}$ (500 $\mathrm{g}$ et $250 \mathrm{~g}$ d'orge/animal/jour, respectivement). 
Tableau I. Ingestion, digestibilité et valeur énergétique des rations, mesurées en sem 11 (moyenne et écart type, 4 brebis par régime).

Niveau d'orge 1

H

$B$

Ingestion de matière sèche (MS)

g/j

$\mathrm{g} / \mathrm{j} / \mathrm{kg} P \mathrm{P}^{0,75}$

$\%$ orge

Digestibilité (\%)

Matière sèche

Matière organique

Énergie de la paille ${ }^{2}$

Valeur énergétique de la ration ${ }^{3}$

$$
\begin{gathered}
1253 \pm 169 \\
64,3 \pm 7,2 \\
37,4 \pm 4,8^{* *}
\end{gathered}
$$

$63,7 \pm 3,2$

$65,1 \pm 3,3$

$49,2 \pm 3,4$

$1,29 \pm 0,09^{*}$
$1199 \pm 116$

$64,0 \pm 9,0$

$19,4 \pm 1,9$

$59,5 \pm 3,4$

$60,7 \pm 3,4$

$51,0 \pm 3,8$

$1,1 \dot{6} \pm 0,08$

$1500 \mathrm{~g}(\mathrm{H})$ ou $250 \mathrm{~g}(\mathrm{~B}) /$ animal/j. ${ }^{2}$ Calculée à partir de la digestibilité estimée de l'énergie de la ration totale, et de la digestibilité théorique de l'énergie de l'orge (INRA, 1988). ${ }^{3}$ Mcal d'énergie nette de lactation (ENI)/kg MS, calculée à l'aide des équations INRA (1988), à partir de la valeur énergétique estimée de la paille et du pourcentage d'orge dans la ration. ", "* Effet du niveau d'orge significatif aux seuils $P<0,10\left({ }^{*}\right)$ ou $P<0,01\left(^{* *}\right)$.

\begin{tabular}{|c|c|c|c|c|c|c|}
\hline \multirow{2}{*}{$\begin{array}{l}\text { Semaines postsevrage } \\
\text { Niveau d'orge }^{2}\end{array}$} & \multicolumn{2}{|c|}{1} & \multicolumn{2}{|c|}{10} & \multicolumn{2}{|c|}{2 à 10} \\
\hline & $H$ & $B$ & $H$ & $B$ & $H$ & $B$ \\
\hline \multicolumn{7}{|l|}{ MS ingérée } \\
\hline$g / j$ & $\begin{array}{l}1235^{* *} \\
\pm 0\end{array}$ & $\begin{array}{l}1002 \\
\pm 0\end{array}$ & $\begin{array}{l}1426 \\
\pm 143\end{array}$ & $\begin{array}{r}1425 \\
+143\end{array}$ & $\begin{array}{r}1427 \\
\pm 138\end{array}$ & $\begin{array}{l}1450 \\
\pm 156\end{array}$ \\
\hline $\mathrm{g} / \mathrm{j} / \mathrm{kg} P \mathrm{PV}^{0,75}$ & $\begin{array}{l}73^{\star *} \\
\pm 5\end{array}$ & $\begin{array}{l}59 \\
\pm 3\end{array}$ & $\begin{array}{l}74 \\
\pm 5\end{array}$ & $\begin{array}{l}76 \\
\pm 6\end{array}$ & $\begin{array}{l}79 \\
\pm 6\end{array}$ & $\begin{array}{l}81 \\
\pm 8\end{array}$ \\
\hline$\%$ orge & $\begin{array}{l}38^{* *} \\
\pm 0\end{array}$ & $\begin{array}{l}23 \\
\pm 0\end{array}$ & $\begin{array}{l}33^{* *} \\
\pm 3\end{array}$ & $\begin{array}{l}17 \\
\pm 2\end{array}$ & $\begin{array}{l}33^{* *} \\
\pm 3\end{array}$ & $\begin{array}{l}16 \\
\pm 2\end{array}$ \\
\hline Énergie ingérée ${ }^{3}$ & $\begin{array}{l}96^{* *} \\
\pm 6\end{array}$ & $\begin{array}{l}71 \\
\pm 4\end{array}$ & $\begin{array}{l}95^{\star *} \\
\pm 5\end{array}$ & $\begin{array}{l}86 \\
\pm 6\end{array}$ & $\begin{array}{l}100^{*} \\
\pm 6\end{array}$ & $\begin{array}{l}92 \\
\pm 8\end{array}$ \\
\hline Énergie disponible 4 & $\begin{array}{l}657^{\star *} \\
\pm 60\end{array}$ & $\begin{array}{l}221 \\
\pm 53\end{array}$ & $\begin{array}{l}715^{\star \star} \\
\pm 106\end{array}$ & $\begin{array}{l}539 \\
\pm 120\end{array}$ & $\begin{array}{l}780^{*} \\
\pm 105\end{array}$ & $\begin{array}{l}612 \\
\pm 142\end{array}$ \\
\hline
\end{tabular}

Tableau II. Ingestion et apports énergétiques durant les différentes phases de la période expérimentale (moyenne et écart-type, 9 brebis par régime).

${ }^{1}$ Moyennes sur $5 \mathrm{j}$ (sem 1), sur $7 \mathrm{j}$ (sem 10) ou sur $64 \mathrm{j}$ (sem 2 à 10). ${ }^{2} 500 \mathrm{~g}(\mathrm{H})$ ou $250 \mathrm{~g}\left(\mathrm{~B}\right.$ )/animal/j. ${ }^{3} \mathrm{Kcal}$ d'énergie nette de lactation (ENI)/j/kg PV $0,75 .{ }^{4} \mathrm{Kcal}$ d'ENI disponible au-dessus des besoins d'entretien par $\mathrm{j}$ et par animal. *, ** Effet du niveau d'orge significatif aux seuils $P<0,05\left({ }^{*}\right)$ ou $P<0,01\left(^{* *}\right)$. 
semaine 1 de l'essai, où l'offre de paille était limitée (tableau II).

\section{Variations du poids vif (PV), de l'état corporel (EC), de la cellularité et de la composition chimique du tissu adipeux mésentérique}

Le niveau de supplémentation en orge n'a pas modifié significativement le gain de PV ou l'accroissement de la note d'EC pendant les 10 sem d'expérience. L'utilisation d'une ration de paille, ad libitum, et de 250 ou $500 \mathrm{~g}$ par j d'orge ont permis des augmentations moyennes d'environ $7 \mathrm{~kg}$ de PV et 0,5 points d'EC (tableau III).

Le niveau de supplémentation en orge n'a aucun effet significatif ni sur la compo- sition chimique du tissu adipeux mésentérique, ni sur la taille de ses adipocytes au terme des $10 \mathrm{sem}$ d'expérience. En revanche, on observe des variations significatives de ces paramètres entre le début et la fin de la période expérimentale (tableau III) : le volume des adipocytes s'accroît d'environ 1,9 fois, et la teneur en lipides d'environ 0,5 fois. Le nombre d'adipocytes par $g$ de tissu a donc été sensiblement réduit, de 12 à 3 millions.

Pour l'ensemble des 36 mesures, on observe une corrélation significative $(r=$ $0,79, P<0,001$ ) entre le volume moyen des adipocytes et la note d'EC des brebis au moment de biopsies (fig 2). Cependant, une corrélation plus élevée $(r=0,84, P<0,001)$ est observée entre le volume des adipocytes et la quantité totale de lipides dans le

Tableau III. Variations du poids vif, de l'état corporel et de l'adiposité ${ }^{1}$ pendant la période expérimentale ( 9 brebis par régime).

\begin{tabular}{|c|c|c|c|c|c|c|}
\hline Période (sem) & & État ini & $i a)^{1}$ & & iation (1 & \\
\hline Niveau d'orge ${ }^{2}$ & $H$ & $B$ & $E T^{3}$ & $H$ & $B$ & $E T^{3}$ \\
\hline $\begin{array}{l}\text { Poids vif }(\mathrm{kg}) \\
\text { Note d'état corporel (NEC) }\end{array}$ & $\begin{array}{r}45,3 \\
1,9\end{array}$ & $\begin{array}{r}43,4 \\
1,9\end{array}$ & $\begin{array}{l}3,3 \\
0,15\end{array}$ & $\begin{array}{l}+6,6^{\mathrm{a}} \\
+0,6^{\mathrm{a}}\end{array}$ & $\begin{array}{l}+6,8^{a} \\
+0,4^{a}\end{array}$ & $\begin{array}{l}2,0 \\
0,1\end{array}$ \\
\hline $\begin{array}{l}\text { Tissus adipeux estimés }{ }^{4} \\
\text { Totaux (kg) } \\
\text { Mésentérique (g) }\end{array}$ & $330^{1,9}$ & $320^{1,8}$ & $\begin{array}{l}0,4 \\
48\end{array}$ & $\begin{array}{l}+1,7^{a} \\
+198^{a}\end{array}$ & $\begin{array}{l}+1,4^{a} \\
+159^{a}\end{array}$ & 34 \\
\hline $\begin{array}{l}\text { Tissu adipeux mésentérique } \\
\text { Matière sèche (\%) } \\
\text { Lipides (\%) } \\
\text { Lipides (g) }\end{array}$ & $\begin{array}{r}57 \\
45 \\
156\end{array}$ & $\begin{array}{r}64 \\
51 \\
165\end{array}$ & $\begin{array}{l}17 \\
16 \\
67\end{array}$ & $\begin{array}{l}+28^{\mathrm{a}} \\
+26^{\mathrm{b}} \\
+224^{\mathrm{a}}\end{array}$ & $\begin{array}{l}+19^{\mathrm{a}} \\
+22^{\mathrm{a}} \\
+182^{\mathrm{a}}\end{array}$ & $\begin{array}{l}14 \\
14 \\
36\end{array}$ \\
\hline $\begin{array}{l}\text { Volume moyen des adipocytes (pl) } \\
\text { Nombre de cellules (millions/g de tissu) }\end{array}$ & $\begin{array}{l}82 \\
9,9\end{array}$ & $\begin{array}{l}123 \\
14,0\end{array}$ & $\begin{array}{l}101 \\
11,9\end{array}$ & $\begin{array}{l}+235^{\mathrm{a}} \\
-6,9^{\mathrm{b}}\end{array}$ & $\begin{array}{l}+146^{\mathrm{a}} \\
-10,6^{\mathrm{b}}\end{array}$ & $\begin{array}{l}81 \\
9,7\end{array}$ \\
\hline
\end{tabular}

1 Les biopsies ont été effectuées à la fin des sem 1 et $10 .{ }^{2} 500 \mathrm{~g} \mathrm{(H)}$ ou $250 \mathrm{~g} \mathrm{(B)/animal/j.}{ }^{3}$ Écart type poolé. ${ }^{4}$ Estimés à partir de la note d'état corporel, d'après les équations de Costa-Teixeira (1987) : gras total (GT, kg) : Log GT = 2, 66 Log NEC - 0,49; gras mésentérique (GM, g) : $\log G M=1,91 \log N E C+1,97 .{ }^{5}$ Poids estimé du tissu adipeux mésentérique multiplié par sa teneur en lipides mesurée. ${ }^{a}$ b Variation entre les sem 1 et 10 significativement différente de 0 , aux seuils $P<0,01^{\text {b }}$ ou $P<0,001^{\text {a. }}$. 
tissu adipeux mésentérique, estimée à partir de la note d'état corporel et de la teneur en lipides mesurée dans ce tissu (fig 2).

\section{Activités des enzymes lipogéniques du tissu adipeux mésentérique}

L'augmentation du niveau de supplémentation en orge s'est exclusivement accompagnée d'un accroissement significatif des activités de I'EM et de la G3PDH pour la sem 1 , activités rapportées au gramme de tissu adipeux mésentérique (tableau IV). À la fin de la période expérimentale (sem 10), les activités de toutes les enzymes, sauf celle de la FAS qui présente toujours des niveaux d'activité très faibles, sont significativement inférieures à celles mesurées au début de la période chez les animaux du lot $\mathrm{H}$.

Quand l'activité est exprimée par million d'adipocytes, les animaux du lot $\mathrm{H}$ présentent des niveaux d'activité de l'EM, de la

Tableau IV. Activités enzymatiques ${ }^{1}$ du tissu adipeux (TA) mésentérique au début et à la fin de la période expérimentale ( 9 brebis par régime).

Sem postsevrage

Niveau d'orge ${ }^{2}$
1

$\begin{array}{lll}H & B & E T^{3}\end{array}$

10

$H \quad B \quad E T^{3}$

Par gramme de tissu 4

$\begin{array}{lrrrrrr}\text { LPL } & 34 & 22 & 16 & 18^{\mathrm{a}} & 18 & 9 \\ \text { EM } & 28 & 13 & 9^{* *} & 17^{\mathrm{b}} & 18 & 4 \\ \text { G6PDH } & 226 & 82 & 145 & 93^{\mathrm{c}} & 87 & 53 \\ \text { G3PDH } & 127 & 371 & 515^{* *} & 662^{\mathrm{c}} & 542 & 224 \\ \text { FAS } & 3,4 & 2,2 & 3,1 & 2,3 & 1,6 & 1,7\end{array}$

Par million d'adipocytes 5

$\begin{array}{lrrcrrr}\text { LPL } & 4,5 & 2,8 & 2,2 & 7,2^{\mathrm{a}} & 5,7^{\mathrm{b}} & 3,9 \\ \text { EM } & 3,6 & 2,1 & 1,6^{+} & 7,1^{\mathrm{a}} & 5,7^{\mathrm{a}} & 2,8 \\ \text { G6PDH } & 26,7 & 9,6 & 11,2^{\star \star} & 37,1 & 24,4^{\mathrm{b}} & 15,5 \\ \text { G3PDH } & 136,7 & 58,6 & 57,4^{\star} & 254,8^{\mathrm{b}} & 160,7^{\mathrm{b}} & 86,5^{\star} \\ \text { FAS } & 0,3 & 0,3 & 0,3 & 0,9 & 0,4 & 0,6\end{array}$

Estimées pour le TA mésentérique total ${ }^{6}$

$\begin{array}{lrrrrrr}\text { LPL } & 10,9 & 7,2 & 5,6 & 9,3 & 8,5 & 4,5 \\ \text { EM } & 9,1 & 4,2 & 2,5^{\star \star *} & 9,1 & 8,5^{\mathrm{b}} & 2,2 \\ \text { G6PDH } & 71,5 & 26,7 & 44,0^{*} & 48,7 & 39,7 & 23,7 \\ \text { G3PDH } & 361,1 & 124,2 & 163,5^{* *} & 344,7 & 253,5^{\mathrm{c}} & 98,9_{+} \\ \text {FAS } & 1,0 & 0,7 & 0,9 & 1,2 & 0,7 & 0,8\end{array}$

1 Lipoprotéine-lipase (LPL), enzyme malique (EM), glucose-6-phosphate deshydrogénase (G6PDH), glycérol-3phosphate deshydrogénase (G3PDH) et synthéthase d'acides gras (FAS). ${ }^{2} 500 \mathrm{~g}(\mathrm{H})$ ou $250 \mathrm{~g}(\mathrm{~B}) / \mathrm{animal} / \mathrm{j}$. ${ }^{3}$ Écart type poolé. Effet du niveau d'orge significatif aux seuils $P<0,10\left(^{+}\right), P<0,05\left(^{*}\right)$ ou $P<0,01\left(^{* *}\right) .{ }^{4} \mu$ moles de nucléotides réduits (EM, G6PDH) ou oxydés (FAS, G3PDH) ou d'acides gras libérés (LPL) par h et par g de tissu. ${ }^{5} \mu$ moles par $\mathrm{h}$ et par million d'adipocytes. ${ }^{6}$ D'après le poids estimé du tissu (voir tableau III) et exprimées en mmoles par $\mathrm{h}$ et par tissu. a,b,c Différence entre périodes dans un même lot significative aux seuils $P<0,10$ (c), $P<$ 0,05 (b) ou $P<0,01$ (a). 
Volume des adipocytes (pl)

(a)

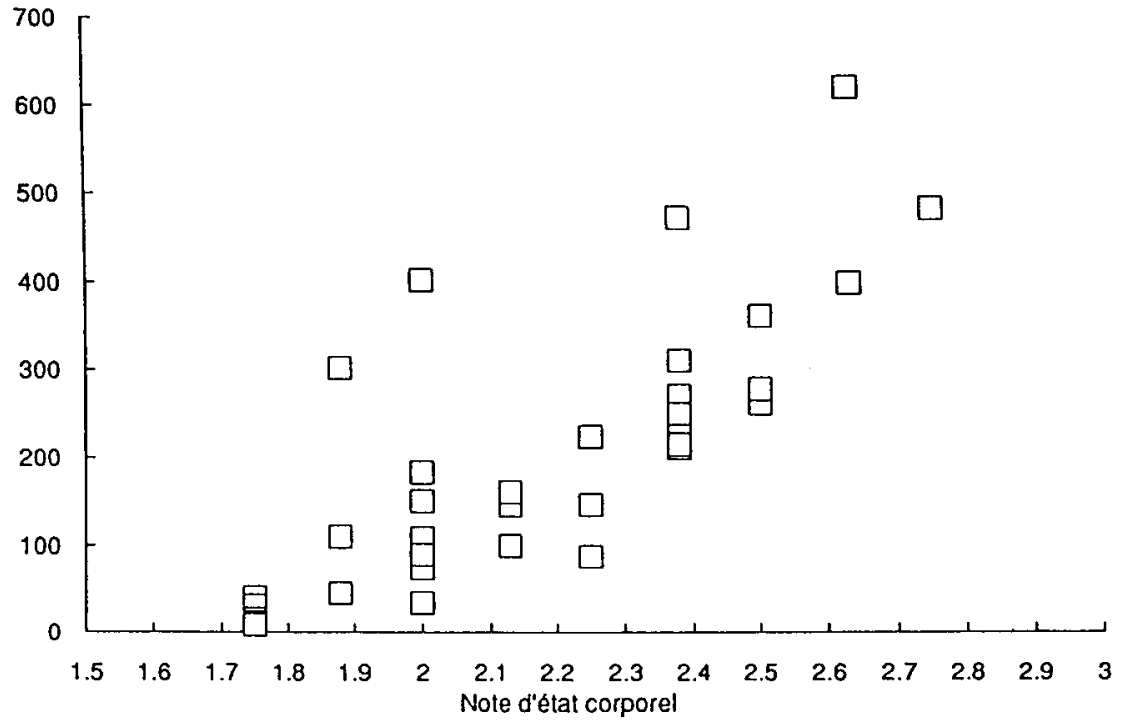

(b)

Volume des adipocytes $(\mathrm{pl})$

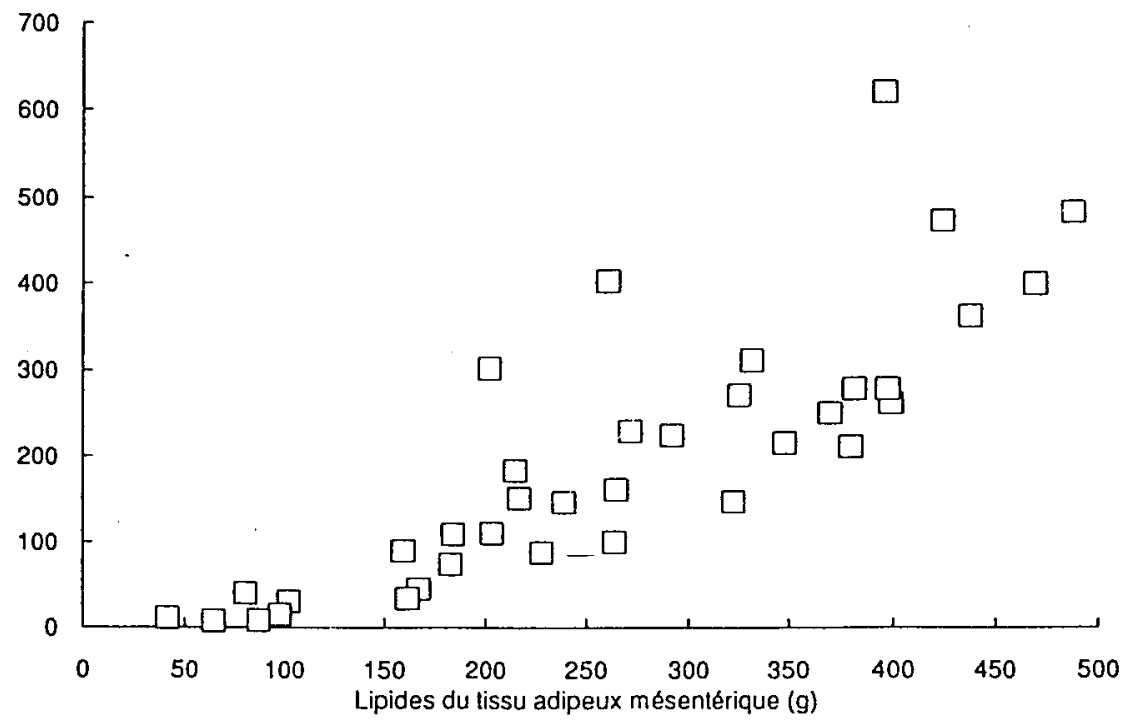

Fig 2. Relations entre le volume (VOL) moyen des adipocytes mésentériques et la note d'état corporel (NEC) (a) et la quantité estimée ${ }^{1}$ de lipides dans le tissu adipeux mésentérique (LIM) (b). 1 Poids du tissu adipeux mésentérique estimé (voir tableau III) multiplié par sa teneur en lipides mesurée. (a) $\mathrm{VOL}(\mathrm{pl})=505 \mathrm{NEC}-896 ; r=0,79$; écart type résiduel $=94 \mathrm{pl}$. (b) $\mathrm{VOL}(\mathrm{pl})=1,22 \mathrm{LIM}(\mathrm{g})-122 ; r=$ 0,84 ; écart type résiduel $=85 \mathrm{pl}$. Les équations sont celles de l'axe principal des régressions $(n=36)$. 
G6PDH et de la G3PDH plus élevés que ceux du lot $B$ en sem 1. Toutes les enzymes (sauf la FAS) ont un niveau d'activité significativement plus élevé à la fin de la période expérimentale. À l'exception de la G3PDH (valeurs moyennes de 255 et 161 $\mu \mathrm{mol} / \mathrm{h} /$ millions d'adipocytes pour les lots $H$ et $B$ en semaine 10 , respectivement, $P<0,05)$, ces augmentations d'activités enzymatiques sont indépendantes du niveau de supplémentation en orge. Les mêmes tendances sont observées quant à l'effet du niveau de supplémentation en orge lorsque les activités sont rapportées au poids total estimé du tissu adipeux mésentérique. En revanche, l'effet "période" est moins apparent (lot B) ou inexistant (lot H).

Pour l'ensemble de la population, les activités enzymatiques du tissu adipeux mésentérique ont été reliées avec l'ingestion d'énergie au moment des biopsies (tableau V). Les corrélations les plus élevées sont observées entre les activités lipogéniques totales estimées du tissu adipeux mésentérique et le bilan énergétique exprimé par $\mathrm{kg}$ de poids métabolique $(r=0,41$ à $0,67, P<0,05$ ) ainsi qu'entre les activités enzymatiques exprimées par million de cellules et l'énergie ingérée par jour ( $r=$ $0,40$ à $0,65, P<0,05)$.

\section{DISCUSSION}

\section{Effet du niveau de supplémentation en orge sur l'ingestion et la digestibilité de la ration}

La diminution de l'apport d'orge a entraîné une augmentation de la quantité de paille ingérée pendant les semaines 2 à 10 de l'expérience (fig 1, tableau II). Le taux moyen de substitution de la paille par le concentré, estimé à 1,2 , est plus élevé que les valeurs théoriques calculées pour ce type de ration à partir des équations proposées par Bocquier et al (1987a) pour des brebis taries ou en lactation. Néanmoins, il est voisin de la valeur de 1,02 observée par Vermorel et al (1987) sur des moutons recevant de la paille traitée à la soude supplémentée par des niveaux de concentré

Tableau V. Coefficients de corrélation $(n=36)$ entre les activités enzymatiques ${ }^{1}$ du tissu adipeux mésentérique et l'ingestion ou le bilan énergétique des brebis.

\begin{tabular}{llllllll}
\hline & \multicolumn{3}{c}{ Énergie ingérée $^{2}$} & & \multicolumn{3}{c}{ Bilan énergetique $^{3}$} \\
\cline { 2 - 3 } Activité enzymatique par : & $\begin{array}{l}\text { Gramme } \\
\text { de tissu }\end{array}$ & $\begin{array}{l}\text { Million de } \\
\text { cellules }\end{array}$ & Tissu & & $\begin{array}{l}\text { Gramme } \\
\text { de tissu }\end{array}$ & $\begin{array}{l}\text { Million de } \\
\text { cellules }\end{array}$ & Tissu $^{4}$ \\
\hline LPL & 0,07 & 0,53 & 0,29 & & 0,30 & 0,34 & 0,41 \\
EM & 0,29 & 0,55 & 0,61 & & 0,52 & 0,30 & 0,67 \\
G6PDH & 0,11 & 0,53 & 0,23 & & 0,37 & 0,48 & 0,46 \\
G3PDH & 0,31 & 0,65 & 0,51 & & 0,56 & 0,47 & 0,67 \\
FAS & 0,09 & 0,40 & 0,24 & & 0,17 & 0,23 & 0,25 \\
\hline
\end{tabular}

1 Lipoprotéine-lipase (LPL), enzyme malique (EM), glucose-6-phosphate déshydrogénase (G6PDH), glycérol-3phosphate déshydrogénase (G3PDH) et synthétase d'acides gras (FAS). ${ }^{2}$ Énergie nette de lactation///animal. ${ }^{3}$ Énergie nette de lactation disponible au-dessus du besoin d'entretien/j/kg PV ${ }^{0,75}{ }^{4}$ Estimée comme indiqué dans les tableaux III et IV. 
voisins de ceux de notre essai (de 5 à $50 \%$ de la MS totale).

Les niveaux d'ingestion de paille observés sur l'ensemble de la période expérimentale (53 et $68 \mathrm{~g} / \mathrm{j} / \mathrm{kg} P \mathrm{PV}^{0,75}$ ) ont été supérieurs à ceux ( 40 à $48 \mathrm{~g}$ ) observés par Faci et al (1988) sur des brebis primipares de la même race et nourries pendant 18 mois avec un régime contenant de 60 à $90 \%$ de paille traitée. Purroy et al (1989) ont également rapporté des valeurs légèrement plus faibles ( 33 à $60 \mathrm{~g}$ ) pendant la période postsevrage sur des brebis adultes de race RA, recevant de la paille traitée et de 0 à $600 \mathrm{~g}$ d'orge par j.

La digestibilité de la matière organique de la ration (dMO) mesurée dans le lot $B$ a été égale à la $\mathrm{dMO}$ calculée additivement à partir des $\mathrm{dMO}$ théoriques des constituants de la ration $(60,7 \%$ et $61,0 \%$ respectivement). En revanche, il est apparu dans le lot $\mathrm{H}$ une légère interaction digestive négative (différence de 2 points, non significative, $P>0,10$ ). Cela a conduit à estimer pour la paille une digestibilité de l'énergie légèrement inférieure dans ce lot à celle estimée pour le lot B $(49,2 \%$ et $51 \%$ respectivement). Ces valeurs sont très proches de celles observées par Vermorel et al (1987) et Alibés et al (1989).

Le fait que l'énergie totale ingérée par le lot $B$ ait été inférieure à celle du lot $\mathrm{H}$ montre que les animaux du lot $B$ n'ont pas réalisé une régulation complète de l'ingestion d'énergie, probablement en raison d'une limitation physique de l'ingestion de paille (Forbes, 1986).

\section{Variations du poids vif, de la note d'état corporel, de la cellularité et de la composition chimique du tissu adipeux mésentérique}

Pendant les 62 jours de lactation qui ont précédé l'expérience, les brebis avaient perdu en moyenne $4 \mathrm{~kg}$ de PV (soit $8 \%$ du $P V$ à la mise bas) et 0,4 point de note d'EC. Au moment du sevrage, le PV et la note d'EC moyens étaient de $44,4 \mathrm{~kg}$ et 1,9 point, respectivement, valeurs insuffisantes pour leur permettre de commencer dans de bonnes conditions un nouveau cycle de reproduction (INRA, 1988).

L'utilisation d'un régime à base de paille, ad libitum, et les 2 niveaux de supplémentation d'orge pendant 9 sem après le sevrage ont permis un niveau d'ingestion d'énergie voisin de 1,8 à 2 fois le niveau d'entretien, et des augmentations moyennes de $6,7 \mathrm{~kg}$ de $\mathrm{PV}$ et 0,5 point de note d'EC. Ces variations ont permis de retrouver (lot $B$ ), voire dépasser (lot $H$ ), le $P V$ et la note d'EC qu'avaient les brebis au moment de l'agnelage. Elles sont légèrement supérieures à celles obtenues dans un essai antérieur $(+4 \mathrm{~kg}$ de PV et $+0,4$ point de note d'EC ; Sebastián et al, 1989) avec un niveau d'alimentation variant de 1,4 à 1,8 fois le besoin d'entretien pendant 12 semaines postsevrage, où le niveau d'alimentation plus faible s'était traduit par une augmentation d'état corporel plus lente que dans la présente expérimentation.

Si on utilise la valeur moyenne de 9,7 kcal par kilogramme de gain de PV proposée par Thériez et al (1987) pour les brebis taries, l'énergie disponible au-dessus de l'entretien aurait dû permettre un gain de PV théorique de $5,1( \pm 0,9)$ et 4,0 $( \pm 0,9) \mathrm{kg}$ pour les lots $\mathrm{H}$ et $B$, respectivement entre les sem 1 et 10 . La différence entre les gains de PV observés et théoriques peut être due à une valeur énergétique inférieure du gain de poids dans le présent essai. Le plus grand écart avec le gain de PV théorique est observé dans le lot $B$, ce qui suggère que, chez ces animaux, la teneur en protéines et/ou en eau du gain de poids est plus importante que chez ceux du lot $H$, en accord avec une tendance (non significative) à une moindre 
accumulation de lipides chez les animaux du lot $B$ (augmentations de la teneur en lipides du tissu adipeux mésentérique de 26 et $22 \%$ et du volume des adipocytes de 235 et 146 pl, pour les lots H et B, respectivement, tableau III). Cette hypothèse est conforme aux schémas déjà proposés de l'évolution de la composition corporelle au cours d'une période de récupération après mobilisation intense des réserves corporelles (Vandermeerschen-Doize et $\mathrm{Pa}$ quay, 1982 ; Sebastián et al, 1989), ainsi qu'avec les différences d'énergie disponible au-dessus des besoins d'entretien dans les 2 lots (tableau II).

Les masses des tissus adipeux totaux et mésentériques n'ont pas été mesurées dans cet essai. Elles ont été estimées à l'aide des équations de Costa-Teixeira (1987) qui relient la note d'état corporel à la quantité de tissus adipeux pouvant être disséqués de brebis taries et non gravides de même race (RA) (tableau III). Le dépôt moyen de tissus adipeux totaux aurait été de 27 et $21 \mathrm{~g}$ par jour chez les animaux des lots $H$ et $B$, respectivement, soit $25 \%$ et $19 \%$ du gain moyen quotidien de PV. II est toutefois possible que ces chiffres sous-estiment le gain effectif de lipides (cf ci-dessous).

La croissance du tissu adipeux des ruminants adultes est due essentiellement au remplissage de la vacuole lipidique des adipocytes existants (hypertrophie). Elle est donc étroitement reliée aux variations de taille des cellules adipeuses (Hood, 1992 ; Robelin et Casteilla, 1990). L'augmentation de la taille des adipocytes du tissu adipeux sous-cutané après le sevrage des brebis semble être plus difficile à mettre en évidence (Hood et Thornton, 1980 ; Sebastián et al, 1989) que celle des tissus adipeux internes (Smith et Walsh, 1984 ; présent essai). Cela peut être lié à des différences d'apports énergétiques entre essais, mais aussi au fait que le tissu adipeux sous-cutané est le dernier à se développer chez les ovins (Russel et al, 1971 ; Teixeira et al, 1989). La diminution apparente du nombre d'adipocytes que l'on peut calculer à partir du poids de tissu adipeux et du nombre d'adipocytes par gramme (tableau III) résulte probablement d'une sous-estimation de l'accroissement de la masse du tissu adipeux mésentérique, du fait que les équations de CostaTeixeira (1987) ont été obtenues sur des brebis de plus petit format que celles de la présente étude.

\section{Activité des enzymes lipogéniques du tissu adipeux mésentérique}

II est préférable d'exprimer les activités métaboliques du tissu adipeux sur une base cellulaire ou tissulaire (tableau IV), car les activités par $\mathrm{g}$ de tissu peuvent varier aussi du seul fait des variations de taille des adipocytes (c'est-à-dire du nombre de cellules par $\mathrm{g}$ de tissu).

La diminution du niveau de supplémentation en orge n'a déterminé des réductions significatives et importantes des activités EM, G6PDH et G3PDH du tissu adipeux mésentérique qu'au début de la période de récupération (sem 1) (tableau IV), lorsque l'offre de paille était limitée et que les animaux n'ont pas pu compenser la différence d'apport d'énergie provenant des céréales (fig 1), ce qui a entraîné une diminution importante de leur bilan énergétique (tableau II).

Des relations positives entre les activités lipogéniques du tissu adipeux et l'ingestion ou le bilan énergétique (tableau $V$ ) ont déjà été rapportées chez les ovins adultes en récupération après une période de sous-alimentation (Ingle et al, 1973; Hood et Thornton, 1980) ainsi que chez la brebis (Vernon et al, 1981 ; Guesnet et al, 1991), la chèvre (Chiliard et al, 1987) et la vache (Chilliard, 1987; Chilliard et al, 
1991) au cours du cycle gestationlactation. Cela reflète une régulation à court terme de l'activité métabolique des tissu adipeux, ne résultant pas des variations à long terme de la taille des adipocytes mais pouvant contribuer à expliquer la tendance à un moindre accroissement de la taille des adipocytes dans le lot $B$ entre les sem 1 et 10 (tableau III).

Les activités de la LPL et de la FAS n'ont pas augmenté significativement avec l'apport d'orge en sem 1. Ces enzymes sont des enzymes régulatrices, et leur activité est généralement limitante (Vernon, 1980) pour le captage des acides gras des triglycérides circulants (LPL) ou la lipogenèse de novo (FAS). II est donc possible que les différences d'état nutritionnel (ou hormonal en découlant) n'aient pas été suffisantes, ou de durée suffisamment longue (Hood et Thornton, 1980), pour induire des modifications significatives de leurs activités. Toutefois, on ne peut exclure que le niveau de sensibilité des méthodes de dosage ne permet pas de mettre en évidence des différences de faible amplitude.

Dans le cas des enzymes non limitantes contrôlant la synthèse du NADPH (EM et surtout G6PDH) et la synthèse du glycérolphosphate pour l'estérification des acides gras (G3PDH), on peut penser au contraire que les variations des concentrations des précurseurs lipogéniques (ou des hormones associées) induites par l'apport supplémentaire d'orge en sem 1 ont été suffisantes pour stimuler rapidement leurs activités, directement ou indirectement, en accord avec les résultats obtenus sur ovins de Ingle et al (1973), Piperova et Pearce (1982), Pearce et Piperova (1985), comparant des rations ayant des teneurs variables en céréales.

En sem 10, les différences d'apport et de bilan énergétique entre les 2 lots sont plus faibles qu'en semaine 1 , et seule l'ac- tivité G3PDH (exprimée par adipocyte ou par tissu) demeure plus élevée dans le lot $H$. Cela peut refléter le fait que l'on observe alors une régulation plus fine au niveau du cycle lipolyse-(ré)estérification des acides gras, avec peu de variations dans les activités de prélèvement et de synthèse des acides gras. Pour les autres enzymes étudiées, il est probable que le niveau d'apport énergétique est plus important que la teneur en orge de la ration pour déterminer le niveau d'activité.

Les activités exprimées par $\mathrm{g}$ de tissu diminuent dans le lot $\mathrm{H}$ entre les sem 1 et 10 , en raison de l'accroisement de la taille des adipocytes, donc du nombre de cellules par g (tableau III). II en résulte que lorsqu'on exprime les activités du lot $\mathrm{H}$ par adipocyte ou par tissu, elles varient peu ou tendent à augmenter pendant la même période, en accord avec la stabilité du bilan énergétique observée entre les sem 1 et 10 dans ce lot (tableau II), et avec les relations positives observées par d'autres auteurs (Hood, 1982) entre les activités lipogéniques et la taille des cellules au cours de la croissance chez les ruminants.

\section{CONCLUSION}

Cette étude a montré qu'une ration à base de paille traitée supplémentée par $250 \mathrm{~g}$ d'orge permet une reconstitution assez rapide des réserves corporelles, lipidiques notamment, chez la brebis tarie. L'accroissement de l'apport d'orge à $500 \mathrm{~g} / \mathrm{j}$ est largement compensé par une réduction de l'ingestion volontaire de paille et n'accroît que faiblement le niveau d'apport énergétique, la note d'état corporel et le volume des adipocytes du tissu adipeux mésentérique, dont les activités des enzymes lipogéniques sont très peu modifiées à long terme, donc peu sensibles à la teneur en céréales de la ration pour un même apport énergétique. 


\section{RÉFÉRENCES}

Alibes X, Muñoz F, Joy M (1989) Aspectos tecnológicos del tratamiento de paja de cereal con urea. Datos preliminares. En : Nuevas Fuentes de alimentos para la producción animal. II. Publ Consejeria de Agricultura y Pesca, Junta de Andalucia, Col Congresos y Jordanas, 12, 287-303

Bocquier F, Theriez M, Brelurut A (1987a) Utilisation du foin par la brebis, 423-455. In: Les fourrages secs : récolte, traitement, utilisation (C Demarquilly, ed). INRA, Paris

Bocquier F, Thériez M, Brelurut A (1987b) Recommandations alimentaires pour les brebis en lactation. Bull Tech CRZV Theix, INRA $70,199-211$

Chilliard Y, Dorléans M, Morand-Fehr P (1977) Mise en évidence d'une activité lipoprotéinelipasique dans le tissu adipeux de chèvre : comparaison de 3 méthodes d'extraction. Ann Biol Anim Biochim Biophys 17, 107-122

Chilliard Y (1987) Revue bibliographique : variations quantitatives et métabolisme des lipides dans les tissus adipeux et le foie au cours du cycle gestation-lactation. 2e partie : chez la brebis et la vache. Reprod Nutr Dév 27, 327-398

Chilliard $Y$, Sauvant $D$, Morand-Fehr $P$, Delouis $C$ (1987) Relations entre le bilan énergétique et l'activité métabolique du tissu adipeux de la chèvre au cours de la première moitié de la lactation. Reprod Nutr Dév 27, 307-308

Chilliard Y, Gagliostro G, Fléchet J, Lefaivre J, Sebastian I (1991) Duodenal rapeseed oil infusion in early and midlactation cows. 5 . Milk fatty acids and adipose tissue lipogenic activities. J Dairy Sci 74, 1844-1854

Costa-Teixeira JA (1987) Reparto de la grasa en función de la condición corporal (body condition) en ovejas adultas Rasa Aragonesa. Tesis Master, IAM-CIHEAM, Zaragosa, España

Cowan RT, Robinson JJ, McDonald I, Smart R (1980) Effects of body fatness at lambing and diet in lactation on body tissue loss, feed intake and milk yield of ewes in early lactation. J Agric Sci Camb 95, 497-514

Faci R, Muñoz F, Alibes X (1988) Resultados productivos en ovejas alimentadas con paja de cereal tratada con amoniaco anhidro. ITEA 78, 45-49
Forbes JM (1986) The voluntary food intake of farm animals. Butterworths and Co Ltd, Londres

Glock GE, McLean P (1953) Further studies on the properties and assay of glucose-6phosphate dehydrogenase and 6phosphogluconate dehydrogenase of rat liver. Biochem J 55, 400-408

Guesnet PM, Massoud MJ, Demarne Y (1991) Regulation of adipose tissue metabolism during pregnancy and lactation in the ewe: the role of insulin. J Anim Sci 69, 2057-2065

Halestrap AP, Denton RM (1973) Insulin and the regulation of adipose tissue acetyl-coenzyme A carboxylase. Biochem J 132, 509-517

Hood RL (1982) Relationship among growth, adipose cell size and lipid metabolism in ruminant adipose tissue. Fed Proc 41, 2555-2561

Hood RL, Thornton RF (1980) The effect of compensatory growth on lipogenesis in ovine carcass adipose tissue. Aust J Agric Res 31, 155-161

Ingle DL, Bauman DE, Mellenberger RG, Johnson DE (1973) Lipogenesis in the ruminant: effect of fasting and refeeding on fatty acid synthesis and enzymatic activity of sheep adipose tissue. J Nutr 103, 1479-1488

INRA (1988) Alimentation des bovins, ovins et caprins. INRA, Paris

Ochoa $S$ (1955) Methods in enzymology, vol 1 (SP Colowick, NO Kaplan, eds). Academic Press, New York, 735

Pearce J, Piperova LS (1985) A comparison of the effects of duodenal glucose infusion on carbohydrate and lipid metabolism in liver, adipose tissue and small intestinaal mucosa in sheep. Comp Biochem Physiol 81B, 59-62

Piperova LS, Pearce J (1982) A comparison of the effects of feeding concentrate diets, based on either maize or barley, or dried grass on adipose tissue lipogenesis in sheep. Int J Biochem 14, 351-354

Purroy A, Jaime C, Muñoz F, Alibes X (1989) Capacidad de ingestion de paja tratada con amoniaco en ovejas Rasa Aragonesa (RA) despues del destete. Relación entre la paja y el concentrado consumidos (resultados preliminares). En : Nuevas Fuentes de alimentos para la producción animal. II. Publ Consejeria de Agricultura y Pesca, Junta de Andalucia, Col : Congresos y Jordanas, 12, 339-340 
Robelin J (1981) Cellularity of bovine adipose tissues: developmental changes from 15 to 65 percent mature weight. $J$ Lipid Res 22 , 452-457

Robelin J, Barboiron C (1988) Système simple d'analyse d'image semi-automatisée : application en histologie à la mesure de longueurs et de surfaces. Cah Tech INRA 18, 7-18

Robelin J, Casteilla L (1990) Différenciation, croissance et développement du tissu adipeux. INRA Prod Anim 3, 243-252

Russel AJF, Gunn RG, Doney JM (1968) Components of weight loss in pregnant hill ewes during winter. Anim Prod 10, 43-51

Russel AJF, Doney JM, Gunn RG (1969) Subjective assessment of body fat in live sheep. J Agric Sci Camb 72, 451-454

Russel AJF, Doney JM, Gunn RG (1971) The distribution of chemical fat in the bodies of Scottish Blackface ewes. Anim Prod 13, 503-509

Sebastian I, Chilliard $Y$, Jaime $C$, Purroy $A$ (1989) Variations du volume des adipocytes et de la note d'état corporel chez la brebis Rasa Aragonesa pendant la lactation et après le tarissement. Ann Zootech 38, 83-90

Smith RW, Walsh A (1984) Effect of lactation on the metabolism of sheep adipose tissues. Res Vet Sci 37, 320-323

Teixeira A, Delfa R, Colomer-Rocher F (1989) Relationships between fat depots and body condition score or tail fatness in the Rasa aragonesa breed. Anim Prod 49, 275-280

Thériez $M$, Bocquier $F$, Brelurut A (1987) Recommandations alimentaires pour les brebis à l'entretien et en gestation. Bull Tech CRZV Theix, INRA 70, 185-197

Vandermeerschen-Doize F, Paquay R (1982) Modifications du poids vif et de la composition corporelle du ruminant adulte en fonction des conditions alimentaires. Rev Agric 2, 1990-2004

Vermorel M, Dulphy JP, Bouvier JC (1987) Energy utilization of sodium hydroxide treated or untreated straw supplemented with protein or concentrates by adult sheep. 1 . Feed intake, digestibility, metabolizability and net energy value. Arch Anim Nutr Berlin 37, 805-821

Vernon RG (1980) Lipid metabolism in the adipose tissue of ruminant animals. Prog Lipid Res 19, 23-106

Vernon RG, Clegg RA, Flint DJ (1981) Metabolism of sheep adipose tissue during pregnancy and lactation. Biochem J 200, 307-314

Vernon RG, Taylor E (1986) Enzymes of adenosine metabolism of sheep adipose tissue: changes in activity with season, pregnancy and lactation. Horm Metab Res 18, 369-373

Vernon RG, Faulkner A, Finley E, Pollock $H$, Taylor E (1987) Enzymes of glucose and fatty acid metabolism of liver, kidney, skeletal muscle, adipose tissue and mammary gland of lactating and non-lactating sheep. J Anim Sci 64, 1395-1411

Wise LS, Green H (1979) Participation of one isozyme of cytosolic glycerophosphate dehydrogenase in the adipose conversion of 3T3 cells. J Biol Chem 254, 273-275 